\title{
Control of extrinsic porosities in linked metal-organic polyhedra gels by imparting coordination-driven self-assembly with electrostatic repul- sion
}

\author{
Zaoming Wang, ${ }^{\dagger, \star}$ Takuma Aoyama, ${ }^{\S}$ Eli Sanchez-Gonzales, ${ }^{\text {a† }}{ }^{\dagger}$ Tomoko Inose,${ }^{\dagger}$ Kenji Urayama,${ }^{\S}$ Shuhei \\ Furukawa* ${ }^{*} \dagger, \dagger$ \\ †Institute for Integrated Cell-Material Science (WPI-iCeMS), Kyoto University, Yoshida, Sakyo-ku, Kyoto 606-8501, Japan \\ *Department of Synthetic Chemistry and Biological Chemistry, Graduate School of Engineering, Kyoto University, Katsura, \\ Nishikyo-ku, Kyoto 615-8510, Japan \\ ${ }^{\S}$ Department of Macromolecular Science and Engineering, Kyoto Institute of Technology, Matsugasaki, Sakyo-ku, Kyoto \\ 606-8585, Japan
}

\begin{abstract}
The linkage of metal-organic polyhedra (MOPs) for the synthesis of porous soft materials is one of the promising strategies to combine the processability with permanent porosity. Compared to the defined internal cavity of MOPs, it is still difficult to control the extrinsic porosities generated between crosslinked MOPs because of their random arrangements in their networks. Herein, we report a method to form linked MOP gels with controllable extrinsic porosities by introducing negative charge on the surface of MOPs that facilitates electrostatic repulsion between them. A hydrophilic rhodium-based cuboctahedral MOP (OHRhMOP) with 24 hydroxyl groups on its outer periphery can be controllably deprotonated to impart the MOP with tunable electrostatic repulsion in solution. This electrostatic repulsion between MOPs stabilizes the kinetically trapped state, in which a MOP is coordinated with various bisimidazole linkers in a monodentate fashion at a controllable linker/MOP ratio. Heating of the kinetically trapped molecules leads to the formation of gels with similar colloidal networks but different extrinsic porosity. This strategy allows us to design the molecular-level networks and the resulting porosities even in the amorphous state.
\end{abstract}

\section{INTRODUCTION}

The development of new porous materials with controllable structures and designable properties is a keystone of modern chemical technology. Metal-organic polyhedra (MOPs), a class of discrete coordination cages with well-defined internal cavities, have emerged as microporous building blocks for the construction of extended porous architectures due to their good solubility and designable outer periphery available for further connection. ${ }^{1-2}$ By crosslinking MOPs with ditopic linkers ${ }^{3-4}$ or tuning the noncovalent interaction between them (hydrogen bonding, ${ }^{5-6}$ electrostatic interaction ${ }^{7-8}$ and host-guest interaction, ${ }^{9-10}$ for instance), various multidimensional assemblies are synthesized, ranging from crystalline porous solids like metal-organic frameworks (MOFs) ${ }^{11}$ to amorphous soft materials such as films ${ }^{12-13}$ or gels. ${ }^{14-16}$ Considering practical applications, the latter has attracted increasing interests because of their potentials for realizing processable microporous materials. ${ }^{17-18}$ For example, MOP-based gels consisting of hierarchical porosities with macro-, meso- and micropores guarantee the transportation of guest molecules throughout the materials, providing an ideal platform for drug release, catalysis, gas separation and rapid sensing. ${ }^{19-20}$ However, in contrast to MOFs with periodically ordered structures that allow for the precise modulation of pore size and shape ${ }^{21}$ it is rather difficult to control these amorphous porous structures. This is because MOP-based amorphous systems possess not only the defined internal cavities but also the irregular extrinsic pores created between randomly arranged or interlinked MOPs. The creation of porous soft materials with high structural controllability and pore designability analogous to MOFs still remains challenging.

By controlling the coordination-driven self-assembly pathway of MOPs, recently we reported the successful fabrication of the linked MOP gels featuring hierarchical porous networks. ${ }^{22-23}$ Instead of forming orderly extended frameworks like MOFs, the random crosslinking of MOPs as junctions with bisimidazole links results in the formation of colloids, followed by their further connections to form colloidal gels. In particular, we showed that the post-synthetic aging process induced the reorganization of crosslinked MOP networks inside the colloids, and thus the control of extrinsic porosity. ${ }^{24}$ However, so far only limited types of linkers have been successfully installed to form the linked MOP gels. In our previous trials to control their extrinsic porosity using flexible links, only spherical particles were obtained instead of colloidal gels. ${ }^{18}$ Considering the importance of linker versatility in the porosity modulation of MOFs, ${ }^{25-26}$ this limitation becomes problematic to the expansion of porous soft materials for potential applications.

Besides the linker versatility, the network topology also dictates the structure of extrinsic porosity. ${ }^{27}$ One of the straightforward ways to control the topology in linked MOP network is 


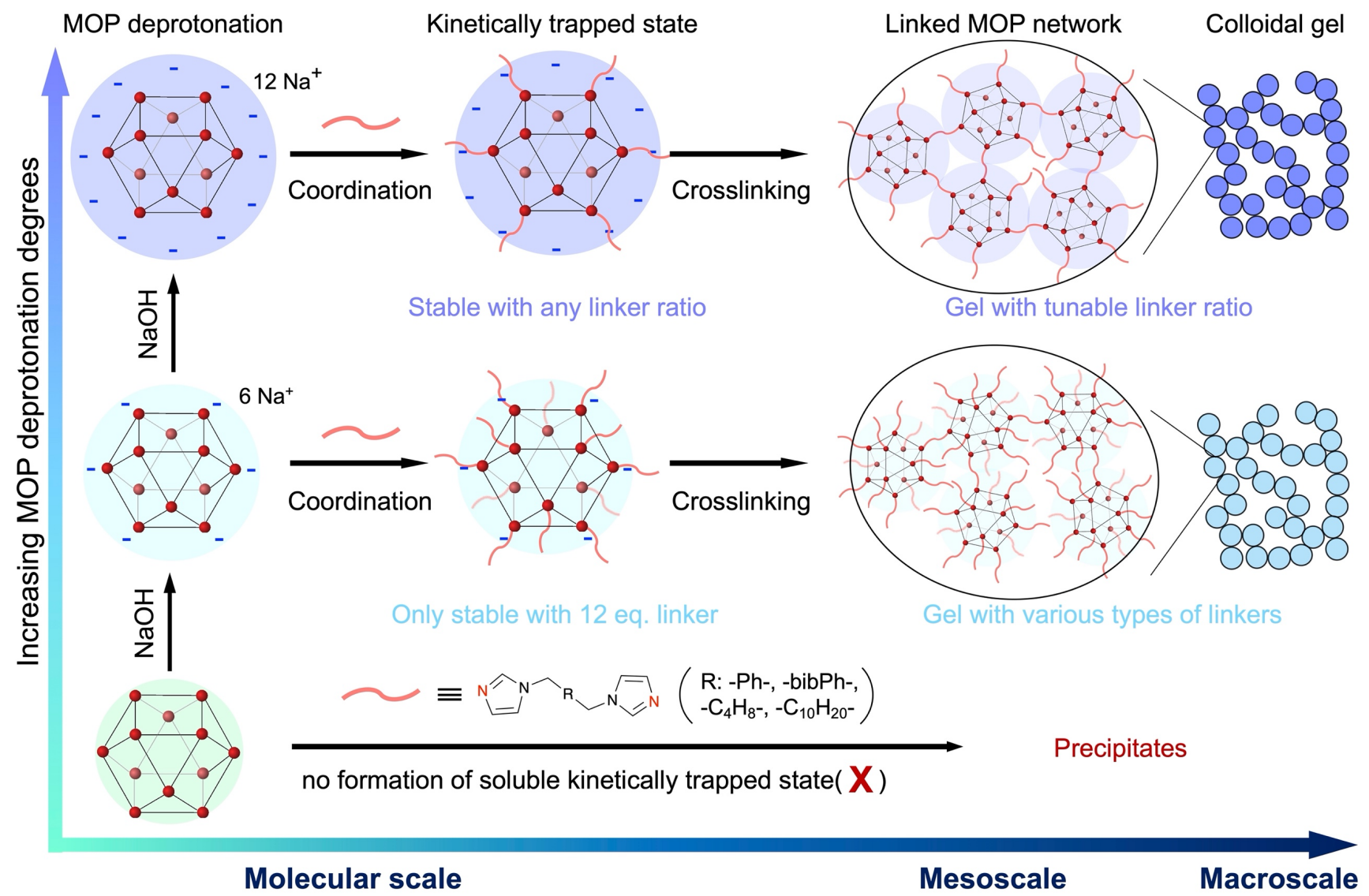

Figure 1. Schematic illustration of the controlled formation of linked MOP gels with tunable linkers types and ratio by using MOPs at different deprotonation degrees.

to change the geometry of MOP and linkers. ${ }^{28-29}$ Another way is to tune the connectivity on the MOP junctions from full crosslinking to partial linkage to thus control the branched functionality of networks. This method is more characteristic of amorphous networks that offer structural freedom within the material. ${ }^{30}$ Although the similar method can be applied to crystalline MOFs, the changing of connectivity on the metal nodes requires the introduction of defects and at the extreme case leads to the collapse of crystalline networks. ${ }^{31-32}$ One of the drawbacks to investigate the amorphous network is to determine the exact network structures and to give feedback to the synthetic conditions. ${ }^{33}$ Though the extrinsic porosity of linked MOP networks can be designed by the linker versatility and the network topology, the appropriate characterization is essential to correlate the synthesis-structure relationship.

Here we report the formation of linked MOP gels with tunable extrinsic porosity by the charge-assisted coordinationdriven self-assembly of a hydrophilic rhodium-based cuboctahedral MOP, $\left[\mathrm{Rh}_{2}(\mathrm{OH}-\mathrm{bdc})_{2}\right]_{12}$ (OHRhMOP, OH-bdc $=$ 5-hydroxyl-1,3-benzenedicarboxylate), with bisimidazole linkers. The presence of 24 hydroxyl (-OH) groups on the MOP periphery can be step-wisely deprotonated in the basic solution to generate controllable native charges on the MOP surface up to -24 charge. This negative charge provides the electrostatic repulsion between MOPs and competes with their linkage by bisimidazole ligand. By balancing these two interactions, a kinetically trapped state, in which each MOP is coordinated by linkers in a monodentate fashion, can be stabilized after mixing with various types of linkers at a controlled ratio. Heating of the kinetically trapped state leads to the formation of linked MOP gels with controlled network connectivity at the MOP node. The nitrogen sorption measurement and the pore size distribution analysis clearly show the influence of linker type and ratio on the structure of extrinsic porosity between MOPs. This approach based on the synergistic effect of attractive and repulsive interactions between MOPs offers a new opportunity to explore the synthesis-structure relationship within amorphous porous networks.

\section{RESULTS AND DISCUSSION}

Influence of MOP charge on coordination-driven crosslinking with bidentate linkers. The cuboctahedral OHRhMOP, composed of 12 rhodium paddlewheel structures and $24 \mathrm{OH}-$ bdc, has a good solubility in polar solvents like alcohols. Once coordinative solvents coordinate to the axial site of OHRhMOP, the molecule can be even soluble in water. We previously demonstrated the dissolution of OHRhMOP in the mixed solvent of water/acetonitrile and the successful formation of linked MOP gels by crosslinking MOPs with a ditopic linker, 1,4-bis(imidazol-1-ylmethyl)benzene (bix).$^{20}$ In general, to form linked MOP gels, the prerequisite is to add 12 equivalent (eq.) of linker bix to a MOP to form so-called kinetically trapped molecule at the chemical composition of $\operatorname{RhMOP}(\text { bix })_{12}$, in which 12 bix molecules coordinate in a monodentate fashion to the 12 available axial sites of rhodium paddlewheels existing on the surface of MOP. On the other hand, the stepwise addition 
of linkers to the MOP solution results in the formation of colloidal particles, named as coordination polymer particles (CPP). To study the effect of MOP charge on the coordination reaction with linkers, titration experiments were performed by stepwise addition of bix solution to the MOP solution at different deprotonation degrees, and the corresponding products were monitored by dynamic light scattering (DLS) measurements (Figure $2 a-b)$.
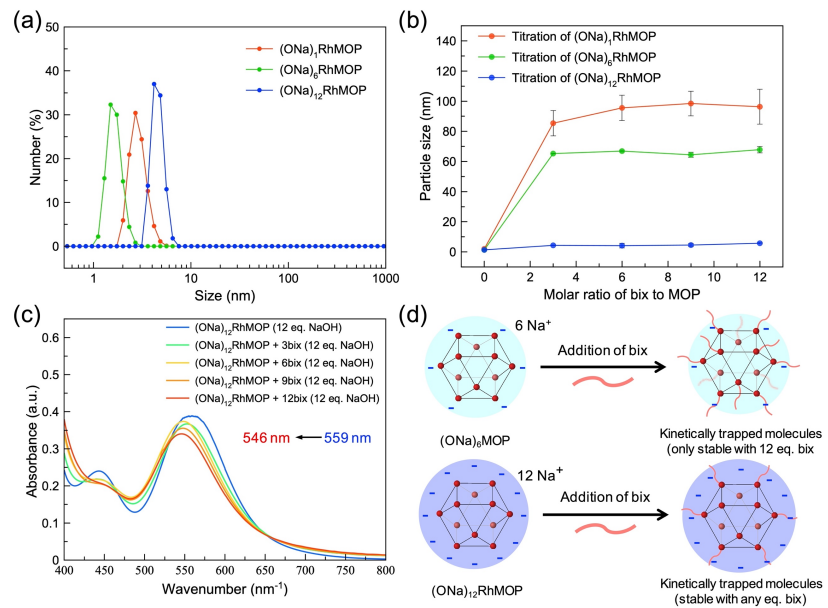

Figure 2. (a) DLS measurement of the MOPs in water/acetonitrile solution at different deprotonation degrees. (b) DLS-determined size evolution of the assembling products obtained during the titration of differently charged MOPs $(0.46 \mathrm{mM})$ by stepwise addition of bix. (c) UV-visible spectroscopy of the (ONa) $)_{12}$ RhMOP solution with different molar ratios of bix. (d) Schematical showing the difference in the formation of kinetically trapped molecules by using MOPs at different deprotonation degrees.

The hydroxyl groups of OHRhMOP were firstly deprotonated in the solution of water/acetonitrile by adding $\mathrm{NaOH}$ to obtain its negatively charged form as $\left[\mathrm{Rh}_{24}(\mathrm{ONa}-\mathrm{bdc})_{n}(\mathrm{OH}-\right.$ bdc $)_{24-n}$, denoted as $(\mathbf{O N a})_{n} \mathbf{R h M O P}$ where $n$ indicates the number of deprotonated -OH groups per MOP. By changing the relative equivalent (eq.) of $\mathrm{NaOH}$ to OHRhMOP for deprotonation, (ONa) ${ }_{n} \mathbf{R h M O P}$ with different charges can be obtained with $\mathrm{n}$ ranging from 1 to 24 , which allows for the control of electrostatic interaction/repulsion between MOPs. The solubility of these deprotonated MOPs in water/acetonitrile was confirmed by DLS measurements showing a number-weighted size distribution around 1-5 nm (Figure 2a). During the titration experiments, the stepwise addition of linker bix $(1 \mathrm{~mol}$. eq. per step) into the solution of (ONa) $)_{1} \mathbf{R h M O P}$ induced obvious growth of its particle size to $85 \pm 8 \mathrm{~nm}$ at the addition of $3 \mathrm{~mol}$. eq. of bix (Figure 2b). This size growth suggests the occurrence of coordination reaction and thus the formation of CPPs. For $(\mathbf{O N a})_{6} \mathbf{R h M O P}$ with a higher charge, a smaller particle size of $65 \pm 1 \mathrm{~nm}$ was obtained after the addition of $3 \mathrm{~mol}$. eq. of bix. Further increasing the deprotonation degree of MOPs to $(\mathrm{ONa})_{12} \mathrm{RhMOP}$ revealed no formation of CPP but a slight size growth to $5.8 \pm 0.3 \mathrm{~nm}$ upon addition of $12 \mathrm{~mol}$. eq. of bix. This slight change can be most likely attributed to the existence of monodentately coordinating bix to the axial site of rhodium paddlewheel moieties on the MOPs. The obvious decrease in particle size by increasing deprotonation degrees indicates the influence of MOP charges on their subsequent coordination reactions with the linker. One plausible reason is that the electrostatic repulsion between the negatively charged MOPs dominates their assembling state and suppresses their crosslinking even with the existence of linker bix (Figure 2d).

To gain deep insights into the coordination environment of the charged (ONa) ${ }_{12} \mathbf{R h M O P}$ with bix in the solution, UVvisible spectroscopy of the MOP solution was measured during the stepwise addition of bix. As shown in Figure 2c, the addition of bix to the MOP solution induced a continuous shift of the maximum adsorption band $\left(\lambda_{\max }\right)$ from $559 \mathrm{~nm}$ to $546 \mathrm{~nm}$ (Figure $\mathrm{S} 1$ ), which is assigned to the $\pi^{*}-\sigma^{*}$ transition of the dirhodium paddlewheel moiety. ${ }^{34}$ The similar shift in $\lambda_{\max }$ was also observed in a control experiment with rhodium acetate $\left[\mathrm{Rh}_{2}(\mathrm{OAc})_{4}\right]$ in the acetonitrile solution; the replacement of 1 eq. of acetonitrile molecule in the $\left[\mathrm{Rh}_{2}(\mathrm{OAc})_{4}(\text { acetonitrile })_{2}\right]$ (a)
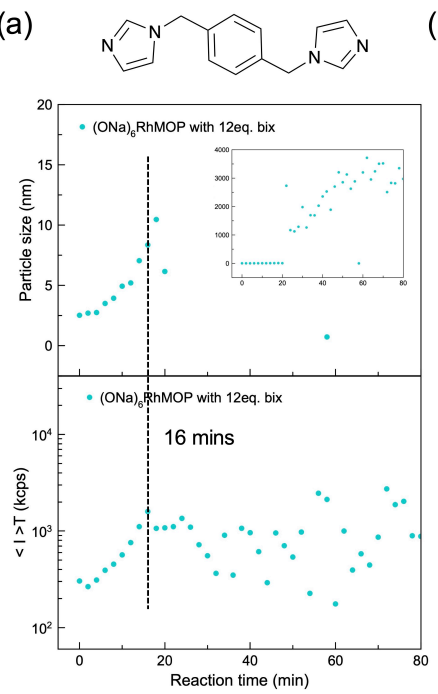

(b)
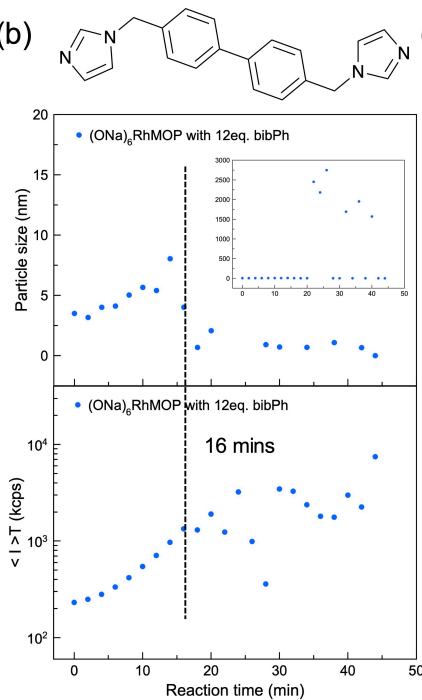

(c)
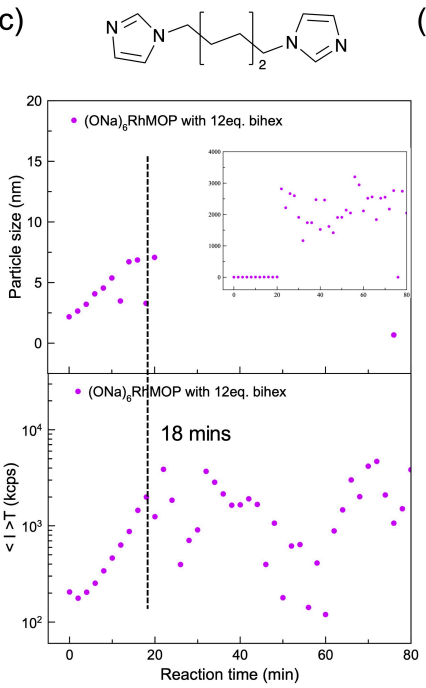

(d)
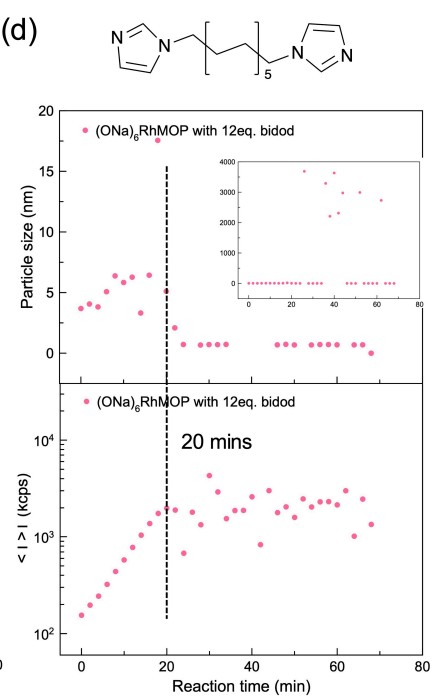

Figure 3. Time-resolved dynamic light scattering (DLS) experiments during the supramolecular polymerization of $(\mathrm{ONa}){ }_{6} \mathrm{RhMOP}$ at $60{ }^{\circ} \mathrm{C}$ at a concentration of $1.4 \mathrm{mM}$, showing the particle size evolution and the time-averaged scattering intensity as a function of times. Systems with 12 molar equivalents of different linkers added to MOPs were measured: (a) bix, (b) bihex, (c) bihex and (d) bidod. 
complex by 1 eq. of monodentate imidazole ligand, 1-dodecyl$1 \mathrm{H}$-imidazole (diz), led to a $\lambda_{\max }$ shift from 552 to $540 \mathrm{~nm}$ (Figure S2). These experiments confirmed the coordination of bix to the outer rhodium sites of MOPs during titration. Combined with the DLS data that revealed no CPP formation at any ratio $(m)$ of bix added, one can hypothesize that only a kinetically trapped molecule, $(\mathbf{O N a})_{12} \mathbf{R h M O P}(\mathbf{b i x})_{m}$ can be formed with monodentately coordinating bix molecules (Figure 2d). Due to the strong electrostatic repulsion, the resulting MOP molecules become more stable and cannot be crosslinked with each other.

MOP gelation with different types of linkers. With the help of stable kinetically trapped molecules, not only bix but other bisimidazole linkers can be installed on the surface of negatively charged MOPs. By implementing the further gelation via heating, it is able to form gels by linking these MOPs, which otherwise are not accessible with the use of their neutral counterparts. As a proof-of-concept, four bidentate linkers with different length and flexibility, including bix, 4,4'-bis(imidazol-1ylmethyl)biphenyl (bibPh), 1,6-di(1H-imidazol-1-yl)hexane (bihex) and 1,12-di(1H-imidazol-1-yl)dodecane (bidod), are used here to crosslink MOPs and to synthesize their corresponding gels (see the molecular formula in Figure S3). In contrast to the solution of $(\mathbf{O N a})_{1} \mathbf{R h M O P}$ that immediately gave precipitates after the addition of bihex and bidod, the addition of 12 mol. eq. of each linker into (ONa) ${ }_{6} \mathbf{R h M O P}$ solution generated stable kinetically trapped molecules in water/acetonitrile solution as $(\mathrm{ONa})_{6} \mathbf{R h M O P}(\mathrm{bix})_{12}, \quad(\mathrm{ONa})_{6} \mathbf{R h M O P}(\mathrm{bihex})_{12}$, $(\mathrm{ONa})_{6} \mathrm{RhMOP}(\mathrm{bibPh})_{12}$ or $(\mathrm{ONa})_{6} \mathrm{RhMOP}(\mathrm{bidod})_{12}$, respectively (Figure S4). To investigate the effect of linker type on the coordination reaction, the crosslinking processes of (ONa) $)_{6} \mathbf{R h M O P}$ with different linkers were monitored by timeresolved DLS experiments at $60^{\circ} \mathrm{C}$, as shown in Figure 3. High temperature is required for the gelation to induce the dissociation of monodentately coordinating linkers from the axial site of MOPs to expose accessible metal sites, to which a neighboring kinetically trapped MOP can coordinate. This coordination reaction sequentially happens to crosslink MOPs. As a result of MOP crosslinking, the particle size in DLS continues to grow until the whole system is frozen by colloidal network formation (Figure 3). To determine the gelation point, the changes in the time-averaged scattering light intensity $\left.(<\mathrm{I}\rangle_{\mathrm{T}}\right)$ are plotted versus the reaction time. The gelation point is associated with the time when the $\langle\mathrm{I}\rangle_{\mathrm{T}}$ starts fluctuation. ${ }^{35}$ Intriguingly, all these four systems showed a similar gelation time of 16-20 mins despite the difference of linkers. This suggests that the polymerization process is predominantly controlled by the MOP charge instead of linker types.

Four self-standing gels with different linkers (bix, bibPh, bihex and bidod) were synthesized by heating the solution of their corresponding kinetically trapped molecules at the same condition $\left(1.4 \mathrm{mM}, 60^{\circ} \mathrm{C}\right)$ (Figure S5). The mechanical properties of the resulting gels, referred to $(\mathbf{O N a})_{6} \mathbf{R h M O P - b i x}$, (ONa) ${ }_{6}$ RhMOP-bibPh, (ONa) ${ }_{6}$ RhMOP-bihex or

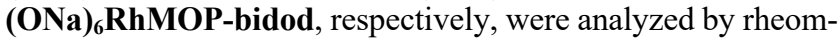
eter in a compression mode. As shown in Figure 4a, all the samples revealed a frequency-independent storage Young's modulus $\left(E^{\prime}\right)$, which was one order of magnitude higher than the loss Young's modulus $(E$ ') $)$. Compared to the gels with more flexible linkers $\left(E^{\prime} \approx 3.7\right.$ and $4.1 \mathrm{kPa}$ for $(\mathbf{O N a})_{6} \mathbf{R h M O P}$-bihex and (ONa) ${ }_{6}$ RhMOP-bidod, respectively), (ONa) ${ }_{6}$ RhMOP-bix and $(\mathbf{O N a})_{6} \mathbf{R h M O P}-b i b P h$ presented higher stiffness with $E$ ' $\approx 6.0$ and $8.0 \mathrm{kPa}$, respectively. Intriguingly, the relative stiffness of the gels also follows the order of linker length; the longer linkers gave the higher stiffness. Then, the order of stiffness of the resulting gels are as follows; $\mathbf{b i b P h}>\mathbf{b i x}>\mathbf{b i d o d}>$ bihex. To further investigate the effect of linker types on the gel structures, all the gel samples were successively exchanged with acetone and dried by supercritical $\mathrm{CO}_{2}$ to obtain their corresponding aerogels for following composition analysis and SEM observation.

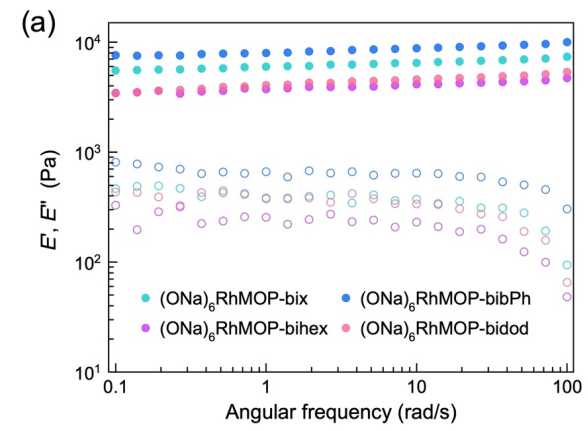

(d)
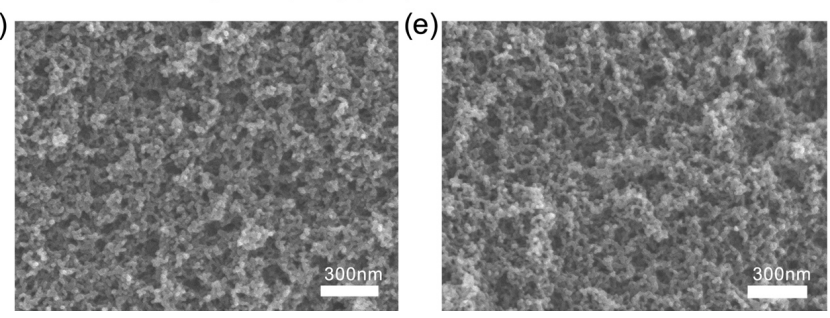
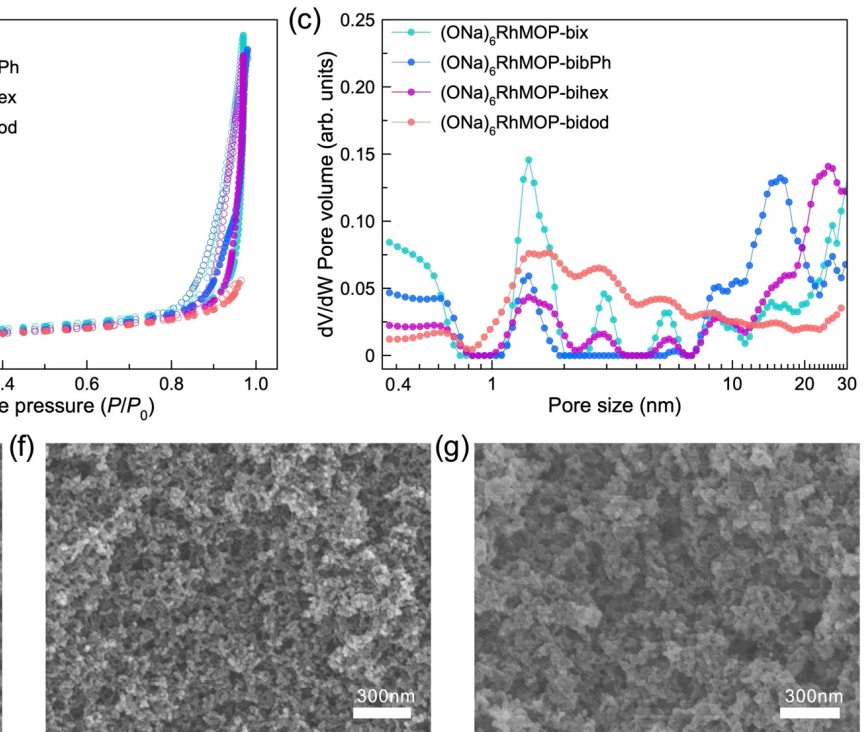

Figure 4. Storage Young's modulus ( $\left.E^{\prime}\right)$ (filled circles) and loss Young's modulus ( $\left.E^{\prime \prime}\right)$ (hollow circles) of gels formed with different linkers versus scanning frequency $(\omega)$. (b) $\mathrm{N}_{2}$ adsorption isotherm at $77 \mathrm{~K}$ for aerogels obtained from (ONa) 6 RhMOP-bix, (ONa) 6 RhMOPbibPh, (ONa) ${ }_{6}$ RhMOP-bihex and (ONa) 6 RhMOP-bidod. (c) The corresponding pore size distribution (PSD) estimated from $\mathrm{N}_{2}$ isotherm by NLDFT on a slit pore model. SEM images of the aerogels obtained from (d) (ONa) 6 RhMOP-bix, (e) (ONa) 6 RhMOPbibPh, (f) (ONa) 6 RhMOP-bihex and (g) (ONa) 6 RhMOP-bidod. 
From ${ }^{1} \mathrm{H}$ NMR experiments of the acid-digested aerogels, the chemical compositions of these four samples were estimated to be $\mathrm{Na}_{6}\left[\mathrm{Rh}_{24}(\mathrm{O}-\mathrm{bdc})_{6}(\mathrm{OH}-\mathrm{bdc})_{18}\right](\mathrm{bix})_{13.1}, \quad \mathrm{Na}_{6}\left[\mathrm{Rh}_{24}(\mathrm{O}-\right.$ bdc $\left.)_{6}(\mathrm{OH}-\mathrm{bdc})_{18}\right](\mathrm{bibPh})_{12.8}, \quad \mathrm{Na}_{6}\left[\mathrm{Rh}_{24}(\mathrm{O}-\mathrm{bdc})_{6}(\mathrm{OH}-\right.$ bdc $\left.)_{18}\right]$ (bihex $)_{13.7}$ and $\mathrm{Na}_{6}\left[\mathrm{Rh}_{24}(\mathrm{O}-\mathrm{bdc})_{6}(\mathrm{OH}-\mathrm{bdc})_{18}\right](\text { bidod })_{13.3}$, respectively (Figure S6-8). The slightly higher linker/MOP ratio $(\approx 13)$ than 12 in each aerogel sample was attributed to the partial MOP decomposition induced by heating of basic solution of MOPs as described in our previous paper. ${ }^{20}$ The scanning electron microscopy (SEM) images of all the aerogel samples revealed similar characteristic colloidal gel networks consisting of interconnected colloidal nanoparticles with the size of around $20 \mathrm{~nm}$ (Figure 4d-g and S9). Based on the similar gelation time and the similar gel compositions and structures among these four samples, we conclude that the gelation process is not influenced by linker types but predominantly controlled by the electrostatic interaction between MOPs. As we already determined that the stiffness of gels is more controlled by the mesoscale structure of colloidal networks rather than the molecular-level structure of crosslinked MOPs, we assume that the difference in the gel stiffness can be explained by the flexibility of linkers at the interface between connected colloids.

The crosslinked MOP structures with different linkers inside each colloidal particle would give more impact on their extrinsic microporosity created between crosslinked MOPs. To understand the relationship between the linker type and the extrinsic microporosity, $\mathrm{N}_{2}$ sorption measurements of these four aerogels obtained from $(\mathrm{ONa})_{6} \mathbf{R h M O P}-\mathbf{b i x},(\mathrm{ONa})_{6} \mathrm{RhMOP}-$ bibPh, (ONa) ${ }_{6}$ RhMOP-bihex and (ONa) 6 RhMOP-bidod were performed at $77 \mathrm{~K}$. As shown in Figure 4b, all the aerogel samples revealed obvious $\mathrm{N}_{2}$ uptake both at the lower relative pressure corresponding to the characteristics of microporosity and at the higher pressure attributed to the capillary condensation of nitrogen inside meso/macro pores, indicating the hierarchical porosity of the resulting aerogels. Based on the sorption isotherm, the corresponding pore size distribution of each aerogel was calculated by nonlocal density functional theory (NLDFT) (Figure 4c) with the slit pore model. For all the samples, a hierarchical pore distribution was observed with the coexistence of multiple pores ranging from nanoscale to mesoscale. Among them, the micropore with the size of ca. 0.6 $\mathrm{nm}$ was attributed to the intrinsic MOP cavity (Figure S12), which confirmed the preservation of most MOP porosities in the gel networks despite their partial decomposition. Three larger pores with the diameter of 1.4, 2.9 and $5.5 \mathrm{~nm}$ were assigned to the extrinsic pores between the crosslinked MOPs, which were clearly observed in the aerogels of (ONa) ${ }_{6}$ RhMOP-bix, (ONa) ${ }_{6}$ RhMOP-bihex. Due to the linker rigidity of bix, (ONa) ${ }_{6} \mathbf{R h M O P - b i x ~ h a s ~ m o r e ~ d e f i n e d ~ e x t r i n s i c ~}$ porosity though bix and bihex has a similar length. On the other hand, $(\mathrm{ONa})_{6} \mathrm{RhMOP}$-bidod showed more broad pores size distribution in the range of $1.4-10 \mathrm{~nm}$. This is more likely attributed to the long and flexible nature of bidod that provides more structural freedom to connect MOPs in different ways. The case for $(\mathbf{O N a})_{6} \mathbf{R h M O P}-\mathbf{b i b P h}$ with long and rigid linkers, however, looks different from others; only the pore at $1.4 \mathrm{~nm}$ can be distinguished while the peaks of pores at 2.9 and $5.5 \mathrm{~nm}$ almost disappeared. In addition, $\mathrm{CO}_{2}$ sorption measurements of all aerogel samples at $196 \mathrm{~K}$ were also performed to reveal a linker-related gas capacity (Figure $\mathrm{S} 11 \mathrm{~b}$ ): at $P / P_{0} \sim 1$, the aerogels obtained from (ONa) ${ }_{6}$ RhMOP-bix, (ONa) ${ }_{6}$ RhMOPbibPh, (ONa) ${ }_{6} \mathrm{RhMOP}-b i h e x$ and $(\mathrm{ONa})_{6} \mathrm{RhMOP}-b i d o d$ adsorbs $181,166,150$ and $143 \mathrm{~cm}^{3}$ of $\mathrm{CO}_{2}$ per gram aerogels, which matched the order of bidod $<$ bihex $<$ bibPh $<$ bix. Combined with the above $\mathrm{N}_{2}$ sorption data, it shows that the extrinsic porosity of the linked MOP gels can be changed by the use of different linkers.
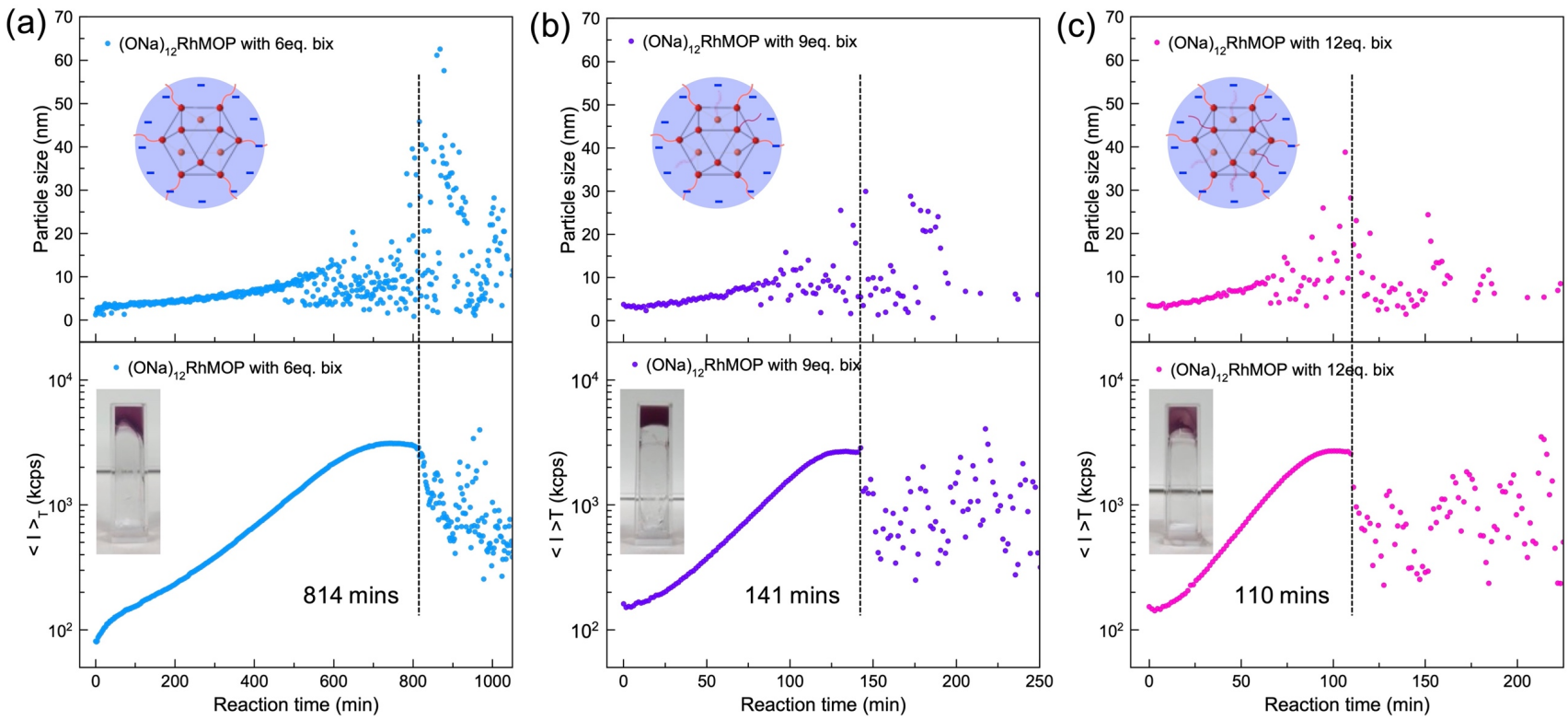

Figure 5. Time-resolved dynamic light scattering (DLS) experiments during the supramolecular polymerization of (ONa) ${ }_{12} \mathbf{R h M O P}$ at $60{ }^{\circ} \mathrm{C}$ at a concentration of $0.46 \mathrm{mM}$, showing the particle size evolution and the time-averaged scattering intensity as a function of times. Systems with different molar equivalents of bix added to MOPs were measured: (a) 6 eq. bix, (b) 9 eq. bix and (c) 6 eq. bix. 


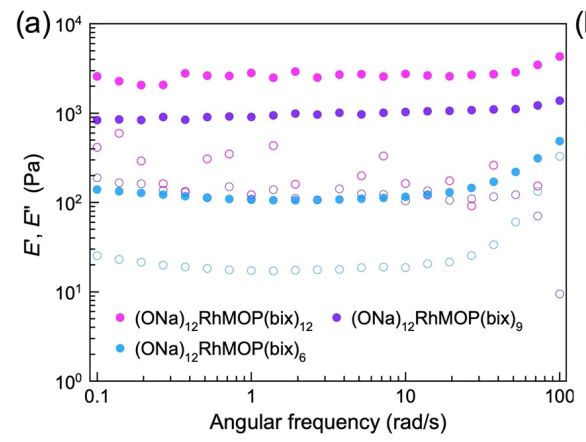

(d)

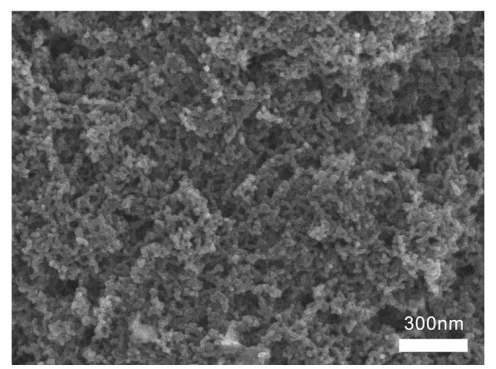

(b)

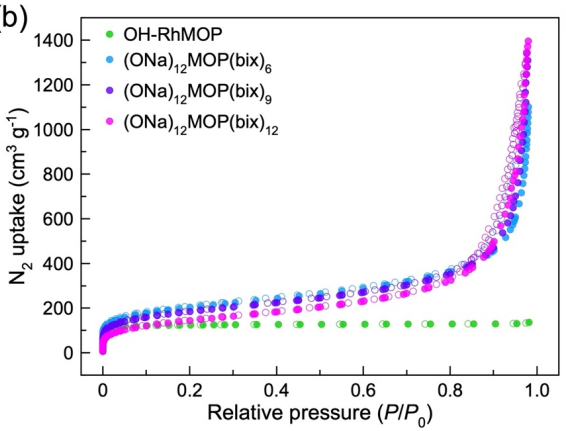

(e)

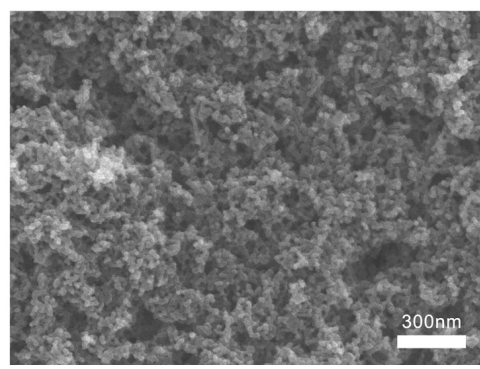

(c)

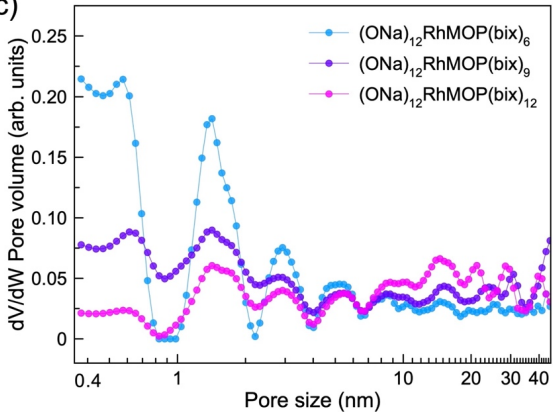

(f)

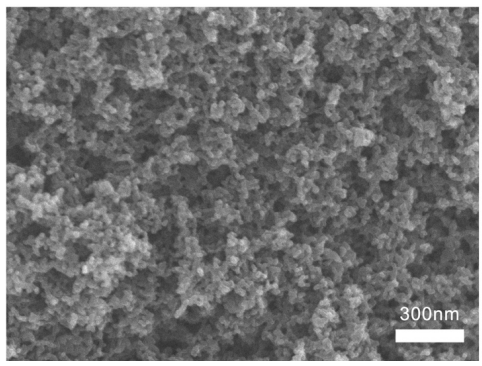

Figure 6. Storage Young's modulus $\left(E^{\prime}\right)$ (filled circles) and loss Young's modulus $\left(E^{\prime \prime}\right)$ (hollow circles) of gels formed with different bix ratio versus scanning frequency $(\omega)$. Note that the $E^{\prime}$ and $E^{\prime \prime}$ of $(\mathbf{O N a})_{12} \mathbf{R h M O P}(\mathbf{b i x})_{\mathbf{6}}$ was estimated from a shearing measurement due to the poor stiffness of this gel which cannot be self-standing (see details in SI). (b) $\mathrm{N}_{2}$ adsorption isotherm at $77 \mathrm{~K}$ for aerogels obtained from $(\mathrm{ONa})_{12} \mathrm{RhMOP}(\mathrm{bix})_{6},(\mathrm{ONa})_{12} \mathrm{RhMOP}(\mathrm{bix})_{\mathbf{9}}$ and $(\mathrm{ONa})_{12} \mathbf{R h M O P}(\mathrm{bix})_{12}$. (c) The corresponding pore size distribution (PSD) estimated from $\mathrm{N}_{2}$ isotherm by NLDFT on a slit pore model. SEM images of the aerogels obtained from (d) (ONa) 12 RhMOP(bix)6, (e) $\left((\mathrm{ONa})_{12} \mathrm{RhMOP}(\mathrm{bix})_{9}\right.$ and (f) $(\mathrm{ONa})_{12} \mathrm{RhMOP}(\mathrm{bix})_{12}$.

Installation of linkers at controlled ratios to obtain gels with different connectivity. To obtain gels in the system of $(\mathbf{O N a})_{6} \mathbf{R h M O P}$, the full coordination of linkers was required to stabilize the kinetically trapped molecule as (ONa) ${ }_{6}$ RhMOP(linker) $)_{12}$. This was the requirement for all the crosslinked MOP gels reported so far. However, in the case of $(\mathbf{O N a})_{12} \mathbf{R h M O P}$, the kinetically trapped molecule can be stabilized with any molar ratio of linker bix thanks to the strong electrostatic repulsion between them (Figure $2 \mathrm{~d}$ ). This stability allows for the control of the crosslinking connectivity of the resulting MOP networks by simply selecting the corresponding linker ratio. To investigate the effect of linker ratio on the polymerization reaction, the crosslinking process of (ONa) ${ }_{12}$ RhMOP with different ratio of bix (6, 9 and $12 \mathrm{~mol}$. eq. relative to MOP, respectively) was monitored by time-resolved DLS experiments at $60^{\circ} \mathrm{C}$. As shown in Figure 5, heating treatment successfully induced the gelation of all solutions but with different gelation times. Determined by the $\langle\mathrm{I}\rangle_{\mathrm{T}}$, the gelation time was found to be strongly influenced by the ratio of bix; the gelation time decreased from 814 mins for MOPs with 6 eq. of bix to 110 mins for MOPs with 12 eq. of bix. This explains that the higher concentration of linker is necessary to induce the crosslinking between highly charged MOPs. The addition of bix less than 6 eq. of bix did not lead to the gelation.

The gels of (ONa) ${ }_{12}$ RhMOP(bix $)_{6}$, $(\mathrm{ONa})_{12}$ RhMOP(bix), and (ONa) ${ }_{12}$ RhMOP(bix) $)_{12}$, were synthesized by adding $6,9,12$ eq. of bix into the solution of (ONa) ${ }_{12}$ RhMOP and then heating at $60{ }^{\circ} \mathrm{C}$ (see details in SI). The corresponding aerogels were synthesized by washing the gels with acetone for three times and then dried by supercritical $\mathrm{CO}_{2}$. To estimate the gel composition, ${ }^{1} \mathrm{H}$ NMR experiments of each aerogel sample after acid-digestion were performed
(Figure S14). For all these three samples, the molar ratio of bix/MOP was estimated to proportional to the amount of bix added; the compositions of gels from $(\mathbf{O N a})_{12} \mathbf{R h M O P}(\mathbf{b i x})_{6}$, $(\mathrm{ONa})_{12}$ RhMOP(bix) $)_{9}$ and $(\mathrm{ONa})_{12} \mathrm{RhMOP}(\mathrm{bix})_{12}$ were $\mathrm{Na}_{12}\left[\mathrm{Rh}_{24}(\mathrm{O}-\mathrm{bdc})_{12}(\mathrm{OH}-\mathrm{bdc})_{12}\right]$ (bix) $)_{8.7}, \quad \mathrm{Na}_{12}\left[\mathrm{Rh}_{24}(\mathrm{O}-\right.$ bdc $\left.)_{12}(\mathrm{OH}-\mathrm{bdc})_{12}\right](\mathrm{bix})_{12.2}$ and $\mathrm{Na}_{12}\left[\mathrm{Rh}_{24}(\mathrm{O}-\mathrm{bdc})_{12}(\mathrm{OH}-\right.$ bdc) $\left.)_{12}\right]$ (bix $)_{14.9}$, respectively. Considering the relatively high deprotonation degree of MOPs, the reason for this higher bix/MOP ratio in gels than it was in the initial kinetically trapped molecules can be attributed to the partial decomposition of MOPs by heating in basic media. Note that the SEM images of these aerogel samples revealed similar colloidal networks despite the difference in their compositions (Figure 6d-f and S15). This similar structure suggested the predominant influence of electrostatic interaction on their gelation process. From the rheological measurements (Figure 6a), the gels exhibited the dependency of mechanical properties on the linker ratio; a lowering the ratio of bix decreases the stiffness of gels. Note that the gel sample of $(\mathbf{O N a})_{12} \mathbf{R h M O P}(\mathbf{b i x})_{6}$ was too weak to be selfstanding (Figure S13a). Therefore, a shearing mode was used to measure its mechanical property (Figure $\mathrm{S} 13 \mathrm{e}$ ) to be $E^{\prime} \approx 0.1$ $\mathrm{kPa}$, which is indeed lower than that of $(\mathbf{O N a})_{12} \mathbf{R h M O P}(\mathbf{b i x})_{9}$ and $(\mathbf{O N a})_{12} \mathbf{R h M O P}(\mathbf{b i x})_{12}$ with $E^{\prime} \approx 0.9$ and $2.8 \mathrm{kPa}$, respectively (to compare the modulus obtained from compression and shearing tests, the rheological data was converted as shown in SI). This significant change in gel stiffness can be ascribed to the higher crosslinking degree between colloidal particles due to the existence of more available bix on their surfaces.

To investigate the effect of linker ratio on the crosslinked MOP structures and thus extrinsic porosity inside each colloidal particle, $\mathrm{N}_{2}$ sorption experiments of the aerogels obtained from $\left.(\mathrm{ONa})_{12} \mathrm{RhMOP}_{(\mathrm{bix}}\right)_{6}, \quad(\mathrm{ONa})_{12} \mathrm{RhMOP}(\mathrm{bix})_{9} \quad$ and 
$(\mathbf{O N a})_{12} \mathbf{R h M O P}(\text { bix })_{12}$ were performed at $77 \mathrm{~K}$ (Figure 6b). Similar to the sorption properties of aerogels discussed above, a hierarchical porous structure was observed in all samples with high $\mathrm{N}_{2}$ uptake at both low and high relative pressure regions. This hierarchical porosity can be further confirmed by their corresponding pore size distribution analysis. In the case of the aerogels obtained from $(\mathbf{O N a})_{12} \mathbf{R h M O P}(\mathbf{b i x})_{6}$, micropores of ca. $0.6 \mathrm{~nm}$ corresponding to internal MOP cavities and the extrinsic pores between crosslinked MOPs at 1.4 and $2.9 \mathrm{~nm}$ are the main contributors for the whole pore volume in the materials. There are less contributions from the larger mesopores. By increasing the bix ratio, we see the trend that the relative contribution from MOP cavities $(0.6 \mathrm{~nm})$ decreases while the contributions from extrinsic porosities (1.4 and $2.9 \mathrm{~nm}$ ) and the mesopores (more than $10 \mathrm{~nm}$ ) increase. This change in the relative pore volume can be attributed to the further crosslinking of MOPs due to the existence of more available bix, leading to higher connectivity at the MOP junctions and thus higher contribution from the extrinsic porosity. As a result, the increasing MOP connectivity inside each colloidal particle contributes to the control of hierarchical porosities in their corresponding aerogels, particularly in the range of extrinsic porosities between crosslinked MOPs and connected colloids. We demonstrated the possibility to design and control the structure and microporosity of soft porous materials even in the amorphous state by the use of the electrostatic repulsion between MOPs.

\section{CONCLUSION}

In summary, we demonstrated a strategy to tune the structures and properties of the linked MOP gels with various types of linkers at a controlled ratio by introducing the electrostatic repulsion between charged MOPs. The outer hydroxyl groups on the MOP periphery were deprotonated by $\mathrm{NaOH}$ to generate the controllable negative charge on the MOPs. The electrostatic repulsion between charged MOPs plays a key role in their following coordination reaction with bisimidazole linkers, especially in the formation of stable kinetically trapped molecules. This stability of kinetically trapped molecules allows us to tune their structure or compositions by simply installing different types of linkers onto MOP surfaces at any ratio without a risk of precipitation through MOP crosslinking. Heating of the kinetically trapped molecules with bisimidazole linkers yielded a series of linked MOP gels with controllable connectivity, mechanical properties and hierarchical porosity. Indeed, the formation of stable kinetically trapped MOP with rationally designed linkers allows us to fabricate versatile self-assembling architectures with controllable porosity. We believe that this strategy is not only limited to the synthesis of linked MOP gels but also applicable to the formation of other MOP-based superstructures with increasing complexity and functionality, such as MOP-based MOFs.

\section{EXPERIMENTAL SECTION}

Materials. Rhodium acetate, ${ }^{34}$ 1,4-bis(imidazol-1-ylmethyl)benzene (bix), ${ }^{18} \quad 4,4$ '-Bis(imidazol-1-ylmethyl)biphenyl (bibPh), ${ }^{18}$ 1,6di(1H-imidazol-1-yl)hexane (bihex), ${ }^{36}$ 1,12-di(1H-imidazol-1-yl)dodecane (bidod) ${ }^{37}$ and OHRhMOP $^{38}$ were synthesized according to a previously reported procedure. 5-hydroxy-1,3-benzenedicarboxylic acid was purchased from Sigma-Aldrich and used as received. Solvents were purchased from Wako Pure Chemical Industries except those at HPLC grade were purchased from Fischer Chemicals.
Characterization. The rheological measurements of the gels were made using a stress-controlled AR-G2 rheometer (TA Instruments, New Castle, DE, USA) and a Modular Compact Rheometer MCR 502 (Anton Paar, Graz, Austria). The supercritical $\mathrm{CO}_{2}$ drying process was carried out on SCLEAD-2BD autoclave (KISCO) using supercritical $\mathrm{CO}_{2}$ at $14 \mathrm{MPa}$ and $50{ }^{\circ} \mathrm{C}$. Scanning Electron Microscopy (SEM) of the microstructures of the aerogel samples were observed using a fieldemission scanning electron microscope with a JEOL Model JSM$7001 \mathrm{~F} 4$ system operating at $10 \mathrm{kV}$ and $5 \mathrm{~mA}$ current. ${ }^{1} \mathrm{H}$ NMR spectra were recorded on a Bruker Biospin DRX-600 (600 MHz) spectrometer. For ${ }^{1} \mathrm{H}$-NMR analysis, $5 \mathrm{mg}$ of aerogel sample was digested in a mixture of DMSO- $\mathrm{d}_{6}(750 \mu \mathrm{l})$ and $\mathrm{DCl}(50 \mu \mathrm{l})$. The mixture was then heated at $100{ }^{\circ} \mathrm{C}$ overnight to obtain a yellow solution. $\mathrm{N}_{2}(77 \mathrm{~K})$ and $\mathrm{CO}_{2}(195 \mathrm{~K})$ gas sorption isotherms of the MOPs and aerogels were recorded on a BELSORP-max volumetric adsorption instrument from BEL Japan Inc. Prior to gas sorption measurement, the samples were activated at $120^{\circ} \mathrm{C}$ for $12 \mathrm{~h}$. UV-visible spectroscopy of the MOP solutions was performed in a V-670 spectrophotometer (JASCO). Timeresolved dynamic light scattering (TRDLS) experiments of the MOP solutions were performed on a Zetasizer Nano ZS instrument (Malvern Instruments, Malvern, UK). The light source was a HeNe laser working at $\lambda=633 \mathrm{~nm}$. The observations were made at the backscattering angle $\theta=173^{\circ}$. The time dependence of particle size and the time-averaged scattering intensity during the gelation process was evaluated at $60^{\circ} \mathrm{C}$.

Titration of MOP-based assemblies by dropwise addition of bix. OHRhMOP was dissolved in a mixture of water and acetonitrile (7:5 $\mathrm{v} / \mathrm{v})$ to obtain a clear purple solution $(0.46 \mathrm{mM}$ MOPs). Then a certain amount of $\mathrm{NaOH}$ (1, 6 and $12 \mathrm{~mol}$. eq. relative to MOP, respectively) was added for deprotonation at different degrees. After sonication, this purple solution was titrated with $0.6 \mathrm{~mL}$ water/acetonitrile solution of $9.2 \mathrm{mM}$ bix at a rate of 1 mol. eq. $(50 \mu \mathrm{L})$ per 5 mins until $12 \mathrm{~mol}$. eq. of bix was added. During the titration, the size change of the MOP assemblies was detected in situ by DLS.

Synthesis of MOP-based gels based on different $(\mathrm{ONa})_{12} \mathrm{RhMOP} / \mathrm{bix}$ ratio. OHRhMOP was dissolved in a mixture of water and acetonitrile $(7: 5 \mathrm{v} / \mathrm{v})$ to obtain a clear purple solution $(2.80$ $\mathrm{mM}$ MOPs). Then $\mathrm{NaOH}$ (12 mol. eq. relative to MOP) was added into the solution for deprotonation. After sonication, $1 \mathrm{~mL}$ water/acetonitrile solution of bix at a certain concentration $(16.8,25.2$ and $33.6 \mathrm{mM}$, respectively) was added to $1 \mathrm{~mL}$ purple solution of $(\mathbf{O N a})_{12} \mathbf{R h M O P}$ under vigorous stirring. The obtained clear purple solution $(1.40 \mathrm{mM}$ MOP) was then placed into a preheated oven at $60{ }^{\circ} \mathrm{C}$ overnight towards gelation. For $(\mathbf{O N a})_{12} \mathbf{R h M O P}(\mathbf{b i x})_{6}$ systems, more times $(\sim 3$ days) were needed to get the corresponding gels.

Synthesis of hydrogels based on $(\mathrm{ONa})_{6} \mathrm{RhMOP}_{\text {with different }}$ linkers. OHRhMOP was dissolved in a mixture of water and acetonitrile $(7: 5 \mathrm{v} / \mathrm{v})$ to obtain a clear purple solution $(2.80 \mathrm{mM})$. Then $\mathrm{NaOH}$ (6 mol. eq. relative to MOP) was added into the solution for deprotonation. After sonication, this purple solution of $(\mathbf{O N a})_{6} \mathbf{R h M O P}$ was added to the water/acetonitrile solution of linker (bix, bibPh, bidod or bihex, $33.6 \mathrm{mM}, 12 \mathrm{~mol}$. eq. for each) under vigorous stirring. The obtained clear purple solution (1.40 mM MOP) was then placed into a preheated oven at $60^{\circ} \mathrm{C}$ overnight towards gelation. After gelation, the gel sample was soaked within fresh water/acetonitrile solution twice to remove the residual chemicals. Then the washed gel was immersed in distilled water for 3 days, replacing the solvent with fresh water each day to remove acetonitrile to get the resulting hydrogels, which were named as (ONa) ${ }_{6}$ RhMOP-bix, (ONa) ${ }_{6}$ RhMOP-bibPh, $(\mathrm{ONa})_{6}$ RhMOP-bihex and (ONa) $)_{6}$ RhMOP-bidod.

Synthesis of aerogels from the hydrogel samples. To obtain aerogel, the as-made hydrogel was soaked with acetone for three days, with the acetone replaced each day. Then the solvent-exchanged samples were then dried by supercritical $\mathrm{CO}_{2}$ at $14 \mathrm{MPa}$ and $40{ }^{\circ} \mathrm{C}$ for 90 mins to obtain the aerogel. Prior to sorption measurements, the aerogel sample was activated at $120^{\circ} \mathrm{C}$ under vacuum for $12 \mathrm{~h}$.

\section{ASSOCIATED CONTENT}




\section{Supporting Information}

The Supporting Information is available free of charge. Details for materials synthesis and characterizations, UV-vis spectroscopy, ${ }^{1} \mathrm{H}$ NMR spectrum, SEM images, photo of gels and sorption results. (PDF).

\section{AUTHOR INFORMATION}

\section{Corresponding Author}

* shuhei.furukawa@icems.kyoto-u.ac.jp

\section{Present Addresses}

a Present address: Instituto de Investigaciones en Materiales, Universidad Nacional Autónoma de México, Circuito Exterior s/n, CU, Del Coyoacán, 04510 México D.F., Mexico.

\section{Notes}

The authors declare no competing financial interest.

\section{ACKNOWLEDGMENT}

Z. W. acknowledges the China Scholarship Council (CSC) for the scholarship support. E.S.G. is grateful to the Japan Society for the Promotion of Science (JSPS) for the Post-Doctoral Fellowship. This study was supported by JSPS KAKENHI Grant Number 18H01995 (Kiban B) and 19H04575 (Coordination Asymmetry) for S.F.

\section{REFERENCES}

1. Hosono, N.; Kitagawa, S., Modular Design of Porous Soft Materials via Self-Organization of Metal-Organic Cages. Acc. Chem. Res. 2018, 51 (10), 2437-2446.

2. Grancha, T.; Carné-Sánchez, A.; Zarekarizi, F.; Hernández-López, L.; Albalad, J.; Khobotov, A.; Guillerm, V.; Morsali, A.; Juanhuix, J.; Gándara, F.; Imaz, I.; Maspoch, D., Synthesis of Polycarboxylate Rhodium(II) Metal-Organic Polyhedra (MOPs) and their use as Building Blocks for Highly Connected Metal-Organic Frameworks (MOFs). Angew. Chem. Int. Ed. 2021, 60 (11), 5729-5733.

3. Uchida, J.; Yoshio, M.; Sato, S.; Yokoyama, H.; Fujita, M.; Kato, T., Self-Assembly of Giant Spherical Liquid-Crystalline Complexes and Formation of Nanostructured Dynamic Gels that Exhibit SelfHealing Properties. Angew. Chem. Int. Ed. 2017, 56 (45), 14085-14089.

4. Lal, G.; Derakhshandeh, M.; Akhtar, F.; Spasyuk, D. M.; Lin, J.B.; Trifkovic, M.; Shimizu, G. K. H., Mechanical Properties of a MetalOrganic Framework formed by Covalent Cross-Linking of MetalOrganic Polyhedra. J. Am. Chem. Soc. 2019, 141 (2), 1045-1053.

5. Sutar, P.; Suresh, V. M.; Jayaramulu, K.; Hazra, A.; Maji, T. K., Binder driven self-assembly of metal-organic cubes towards functional hydrogels. Nat. Commun. 2018, 9 (1), 3587.

6. Qin, Y.; Chen, L.-L.; Pu, W.; Liu, P.; Liu, S.-X.; Li, Y.; Liu, X.L.; Lu, Z.-X.; Zheng, L.-Y.; Cao, Q.-E., A hydrogel directly assembled from a copper metal-organic polyhedron for antimicrobial application. Chem. Commun. 2019, 55 (15), 2206-2209.

7. Gosselin, A. J.; Decker, G. E.; Antonio, A. M.; Lorzing, G. R.; Yap, G. P. A.; Bloch, E. D., A Charged Coordination Cage-Based Porous Salt. J. Am. Chem. Soc. 2020, 142 (21), 9594-9598.

8. Le Ouay, B.; Yoshino, H.; Sasaki, K.; Ohtsubo, Y.; Ohtani, R.; Ohba, M., Crystalline assembly of metal-organic polyhedra driven by ionic interactions with polyoxometalates. Chem. Commun. 2021, 57 (42), 5187-5190.

9. Wei, S.-C.; Pan, M.; Fan, Y.-Z.; Liu, H.; Zhang, J.; Su, C.-Y., Creating Coordination-Based Cavities in a Multiresponsive Supramolecular Gel. Chem. Eur. J. 2015, 21 (20), 7418-7427.

10. Nitta, N.; Takatsuka, M.; Kihara, S.-i.; Hirao, T.; Haino, T., SelfHealing Supramolecular Materials Constructed by Copolymerization via Molecular Recognition of Cavitand-Based Coordination Capsules. Angew. Chem. Int. Ed. 2020, 59 (38), 16690-16697.

11. Lee, J.; Kwak, J. H.; Choe, W., Evolution of form in metalorganic frameworks. Nat. Commun. 2017, 8 (1), 14070.

12. Xie, X.-Y.; Wu, F.; Liu, X.; Tao, W.-Q.; Jiang, Y.; Liu, X.-Q.; Sun, L.-B., Photopolymerization of metal-organic polyhedra: an efficient approach to improve the hydrostability, dispersity, and processability. Chem. Commun. 2019, 55 (44), 6177-6180.

13. Yin, J.-F.; Zheng, Z.; Yang, J.; Liu, Y.; Cai, L.; Guo, Q.-Y.; Li, M.; Li, X.; Sun, T. L.; Liu, G. X.; Huang, C.; Cheng, S. Z. D.; Russell, T. P.; Yin, P., Unexpected Elasticity in Assemblies of Glassy SupraNanoparticle Clusters. Angew. Chem. Int. Ed. 2021, 60 (9), 4894-4900.

14. Gu, Y.; Alt, E. A.; Wang, H.; Li, X.; Willard, A. P.; Johnson, J. A., Photoswitching topology in polymer networks with metal-organic cages as crosslinks. Nature 2018, 560 (7716), 65-69.

15. Shao, L.; Hua, B.; Hu, X.; Stalla, D.; Kelley, S. P.; Atwood, J. L., Construction of Polymeric Metal-Organic Nanocapsule Networks via Supramolecular Coordination-Driven Self-Assembly. J. Am. Chem. Soc. 2020, 142 (16), 7270-7275.

16. Sutar, P.; Maji, T. K., Recent advances in coordination-driven polymeric gel materials: design and applications. Dalton Trans. 2020, 49 (23), 7658-7672.

17. Foster, J. A.; Steed, J. W., Exploiting Cavities in Supramolecular Gels. Angew. Chem. Int. Ed. 2010, 49 (38), 6718-6724.

18. Carné-Sánchez, A.; Craig, G. A.; Larpent, P.; Hirose, T.; Higuchi, M.; Kitagawa, S.; Matsuda, K.; Urayama, K.; Furukawa, S., Selfassembly of metal-organic polyhedra into supramolecular polymers with intrinsic microporosity. Nat. Commun. 2018, 9 (1), 2506.

19. Foster, J. A.; Parker, R. M.; Belenguer, A. M.; Kishi, N.; Sutton, S.; Abell, C.; Nitschke, J. R., Differentially Addressable Cavities within Metal-Organic Cage-Cross-Linked Polymeric Hydrogels. J. Am. Chem. Soc. 2015, 137 (30), 9722-9729.

20. Wang, Z.; Craig, G. A.; Legrand, A.; Haase, F.; Minami, S.; Urayama, K.; Furukawa, S., Porous Colloidal Hydrogels Formed by Coordination-Driven Self-Assembly of Charged Metal-Organic Polyhedra. Chem. Asian J. 2021, 16 (9), 1092-1100.

21. Yaghi, O. M.; O'Keeffe, M.; Ockwig, N. W.; Chae, H. K.; Eddaoudi, M.; Kim, J., Reticular synthesis and the design of new materials. Nature 2003, 423 (6941), 705-714.

22. Carné-Sánchez, A.; Craig, G. A.; Larpent, P.; Guillerm, V.; Urayama, K.; Maspoch, D.; Furukawa, S., A Coordinative Solubilizer Method to Fabricate Soft Porous Materials from Insoluble MetalOrganic Polyhedra. Angew. Chem. Int. Ed. 2019, 58 (19), 6347-6350.

23. Legrand, A.; Liu, L.-H.; Royla, P.; Aoyama, T.; Craig, G. A.; Carné-Sánchez, A.; Urayama, K.; Weigand, J. J.; Lin, C.-H.; Furukawa, S., Spatiotemporal Control of Supramolecular Polymerization and Gelation of Metal-Organic Polyhedra. J. Am. Chem. Soc. 2021, 143 (9), 3562-3570.

24. Wang, Z.; Villa Santos, C.; Legrand, A.; Haase, F.; Hara, Y.; Kanamori, K.; Aoyama, T.; Urayama, K.; Doherty, C. M.; Smales, G. J.; Pauw, B. R.; Colón, Y. J.; Furukawa, S., Multiscale structural control of linked metal-organic polyhedra gel by aging-induced linkage-reorganization. Chem. Sci. 2021, 12 (38), 12556-12563.

25. Kitagawa, S.; Kitaura, R.; Noro, S.-i., Functional Porous Coordination Polymers. Angew. Chem. Int. Ed. 2004, 43 (18), 23342375.

26. Horike, S.; Shimomura, S.; Kitagawa, S., Soft porous crystals. Nat. Chem. 2009, 1 (9), 695-704.

27. Gu, Y.; Zhao, J.; Johnson, J. A., Polymer Networks: From Plastics and Gels to Porous Frameworks. Angew. Chem. Int. Ed. 2020, 59 (13), 5022-5049.

28. Zhukhovitskiy, A. V.; Zhong, M.; Keeler, E. G.; Michaelis, V. K.; Sun, J. E. P.; Hore, M. J. A.; Pochan, D. J.; Griffin, R. G.; Willard, A. P.; Johnson, J. A., Highly branched and loop-rich gels via formation of metal-organic cages linked by polymers. Nat. Chem. 2016, 8 (1), 3341. 
29. Sun, Y.; Chen, C.; Stang, P. J., Soft Materials with Diverse Suprastructures via the Self-Assembly of Metal-Organic Complexes. Acc. Chem. Res. 2019, 52 (3), 802-817.

30. Jangizehi, A.; Schmid, F.; Besenius, P.; Kremer, K.; Seiffert, S., Defects and defect engineering in Soft Matter. Soft Matter 2020, 16 (48), 10809-10859.

31. Dissegna, S.; Epp, K.; Heinz, W. R.; Kieslich, G.; Fischer, R. A., Defective Metal-Organic Frameworks. Adv. Mater. 2018, 30 (37), 1704501 .

32. Wu, H.; Chua, Y. S.; Krungleviciute, V.; Tyagi, M.; Chen, P.; Yildirim, T.; Zhou, W., Unusual and Highly Tunable Missing-Linker Defects in Zirconium Metal-Organic Framework UiO-66 and Their Important Effects on Gas Adsorption. J. Am. Chem. Soc. 2013, 135 (28), 10525-10532.

33. Jeyakkumar, P.; Liang, Y.; Guo, M.; Lu, S.; Xu, D.; Li, X.; Guo, B.; He, G.; Chu, D.; Zhang, M., Emissive Metallacycle-Crosslinked Supramolecular Networks with Tunable Crosslinking Densities for Bacterial Imaging and Killing. Angew. Chem. Int. Ed. 2020, 59 (35), 15199-15203.

34. Furukawa, S.; Horike, N.; Kondo, M.; Hijikata, Y.; CarnéSánchez, A.; Larpent, P.; Louvain, N.; Diring, S.; Sato, H.; Matsuda,
R.; Kawano, R.; Kitagawa, S., Rhodium-Organic Cuboctahedra as Porous Solids with Strong Binding Sites. Inorg. Chem. 2016, 55 (21), 10843-10846.

35. Legrand, A.; Craig, G. A.; Bonneau, M.; Minami, S.; Urayama, K.; Furukawa, S., Understanding the multiscale self-assembly of metalorganic polyhedra towards functionally graded porous gels. Chem. Sci. 2019, 10 (47), 10833-10842.

36. Qi, Y.; Luo, F.; Che, Y.; Zheng, J., Hydrothermal Synthesis of Metal-Organic Frameworks Based on Aromatic Polycarboxylate and Flexible Bis(imidazole) Ligands. Cryst.l Growth Des. 2008, 8 (2), 606611.

37. Xu, J.-F.; Chen, Y.-Z.; Wu, L.-Z.; Tung, C.-H.; Yang, Q.-Z., Dynamic Covalent Bond Based on Reversible Photo [4 + 4] Cycloaddition of Anthracene for Construction of Double-Dynamic Polymers. Org. Lett. 2013, 15 (24), 6148-6151.

38. Carné-Sánchez, A.; Albalad, J.; Grancha, T.; Imaz, I.; Juanhuix, J.; Larpent, P.; Furukawa, S.; Maspoch, D., Postsynthetic Covalent and Coordination Functionalization of Rhodium(II)-Based Metal-Organic Polyhedra. J. Am. Chem. Soc. 2019, 141 (9), 4094-4102. 